\title{
Location of Eyes in Images of Human Faces Through Analysis Variance Shine Intensity
}

\author{
Huss ein Alhamzawi \\ Faculty ofInformatics, University of Debrecen \\ Kassai Street 26, 4028 Debrecen, Hungary; phone: +36 304390299
}

\section{Article Info \\ Article history: \\ Received Feb 5, 2018 \\ Revised May 25, 2018 \\ Accepted Jun 14, 2018}

\section{Keywords:}

Binarization

Eye Center Location

Face Detection

Median Filter

\begin{abstract}
Extraction of facial features is an important step in automatic visual interpretation and recognition of human faces. Among the facial features, the eyes play a major role in the recognition process. In this article, we present an approach to detect and locate the eyes in frontal face images. Eye regions are identified using the technique of voucher detection based on mathematical morphology. After this identification is made a comparison between the variances of three different portions of each candidate region to eye (set of pixels belonging to the candidate region as a whole, set of pixels contained in a minimum rectangle circumscribed to the candidate region and set of pixels of the candidate region belonging to a horizontal band that crosses the center of mass of this region). The calculation of these variances also considers the R, G, and B channels, as well as the gray version of the input image.
\end{abstract}

Copyright () 2018 Institute of Advanced Engineering and Science. All rights reserved.

\section{Corresponding Author:}

Hussein Alhamzawi,

Faculty ofInformatics, University of Debrecen,

Kassai Street 26, 4028 Debrecen, Hungary; phone: +36 304390299.

E-mail:Aliov.mezher@g mail.com

\section{INTRODUCTION}

In lasts years ,many applications consider that eye detection is an A decisive step such as detection/recognition of the face, analysis of the facial expression, estimation of the gaze, investigation in the criminal states, surveillance systems, human interactions [1]-[4]. In Existing works, eye detection can be classified into two major groups: A passive approach based on traditional images and Active approach based on infrared.

The former uses the shape of the eye and intensity for detection the latest work on Assuming that the eyes have a reflection under nearby infrared radiation and it produces the effect of a pupil bright / dark. Traditional methods are broadly classified into three categories: Template-based methods [5], [6.] . Appearance-based methods [7], [8] and Feature-based features [9], [10].

Color is a useful feature used in eye detection. Thilak et al. [11] the algorithm by the three levels suggested that detects eyes. Initially, candidates for the eye are selected through a simple threshold on HSV color space and a normal RGB color space sequentially. Then follow it to determine the spatially connected regions by connected component analysis. In the end, applied the variance projection function and mean to check the eye in each window.

In [12] which is used a sliding window and haar-like features, which starts a window revealing the center of the image which actually indicates the eye area to minimize processing time and errors in eye detection. Rubel Biswas, Jia Uddin, Md. Junayed Hasan [13] They used utilized the Daugman's model, after encoding the features DNS and GNS maps [14], [15] Used to reduce the size of the vector features and extract vector element of them IRIS dataset tested. For the purpose of automatic learning at the identification stage, a single layer support vector machine was used. 
In [16] Human face part such as nose, mouth or eye location is important for understanding and recognition the image. Here is a precise modification of HOG Transform to detect eyes and eyelids.

The method proposed in this article is composed of two phases. In the first phase, the candidates for eyes are identified using the algorithm of detection of valleys [17] applied in the images of human faces. Since the iris region has low-intensity gray levels, there will be a valley there. In the second phase, for each eye candidate region, the intensity of its pixels is measured by calculating the variance. Considering that the eye region has at least four distinct colors due to the different "tissues" that make up the region of the eye (skin, sclera, iris, and pupil), it is expected to obtain high variations of brightness. The performance of this method was tested on frontal human face images with ceiling and front illumination.

\section{DETECTION OF EYE CANDIDATES}

The face area was previously detected, so the input image has only the face region. The first step in this method of localization is to obtain the candidates by eye through the algorithm of voucher detection [17]. This algorithm is a sequence of morphological operations applied directly to the image intensity. As the images used in the input were of the RGB type, it was decided, firstly, to test the algorithm on each of the R, $\mathrm{G}$, and $\mathrm{B}$ channels as well as the grayscale image, and it was concluded that the $\mathrm{R}$ channel provided a greater separation of the candidate regions without decreasing the number of pixels of each, especially in the which refers to the regions of the eyes and eyebrows. The Expression below represents the algorithm in question:

$$
V(f)=(f * B)-f
$$

We have $V(f)$ Valley image

$(f * B)$ Morphological closing operation between the input image $f$ and the structuring element $B$.The closure is a dilation followed by erosion using the same element $\mathrm{B}$.

The resulting image (still in gray levels) is binarized by the Otsu method [9], receives a median filtering to exclude is olated pixels and the remaining pixel sets are classified with unique names (connected component-labeling) in order to identify only each candidate region obtained. Thus, the coordinates of the pixels of each candidate region will be able to serve as pointers to the respective regions in the original image, allowing brightness levels to be extracted and analyzed. Figure 1 shows the flowchart of the operations performed at this stage and Figure 2 shows their typical images.

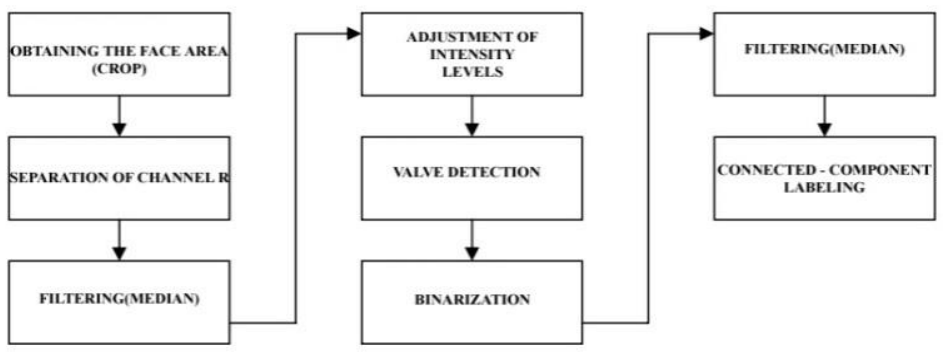

Figure1. Steps of the phase detection candidate regions of the eye

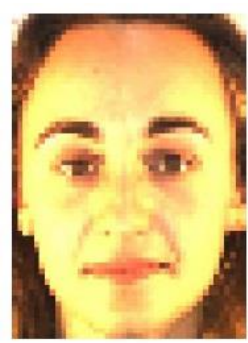

a)

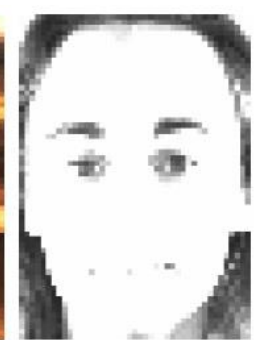

b)

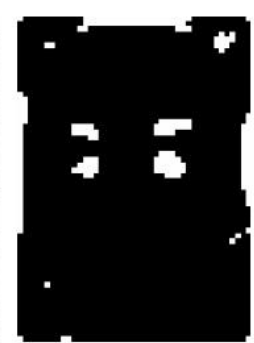

c)

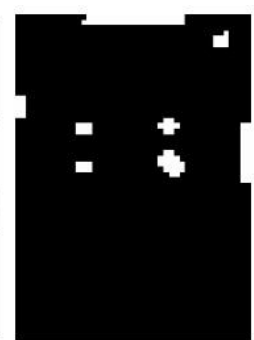

d)

Figure 2. a) Input image, b) R channel, c) Binary-valley image, d) Filtered valley image 


\section{ANALYSIS OF VARIANCE}

At this stage twelve different measures of variance are made in each of the regions detected in the previous phase. That is, for each candidate region three different approaches are given and for each one the variance in the R, G, B channels and the image in gray levels is calculated. These approaches, here, are called portions and are listed:

Portion A: Set of pixels belonging to the candidate region as a whole;

Portion B: Set of pixels contained in a minimum rectangle circumscribed to the candidate region (may contain candidate region pixels and external pixels);

Portion C: Only the set of pixels of the candidate region belonging to a horizontal band that crosses the center of mass of this region.

The portions $\mathrm{A}$ and $\mathrm{B}$ were chosen for obvious reasons and the portion $\mathrm{C}$ to be in the direction which is supposedly the most variation in color of the tissues that comprise the human eye. In all three cases, the pixels belonging to the portions were arranged in one-dimensional vectors and the variance calculation was applied.

$$
S^{2}=\frac{\sum_{i=1}^{n}\left(x_{i}-\bar{x}\right)^{2}}{(n-1)}
$$

To obtain the center of mass $(m x, m y)$ of the candidate region in order to extract the portion $\mathrm{C}$, the following expressions were used:

$$
\begin{aligned}
& m x=\operatorname{int}\left(\frac{1}{N_{b}}\left(\sum_{i=1}^{M} \sum_{j=1}^{N} i f_{b}(i, j)\right)\right. \\
& m y=\operatorname{int}\left(\frac{1}{N_{b}}\left(\sum_{i=1}^{M} \sum_{j=1}^{N} i f_{b}(i, j)\right)\right.
\end{aligned}
$$

\section{EXPERIMENTAL RESULT}

The data of our testing was taken from the students at the University of Debrecen, Hungary and from some people around the university the data set was captured in bright light only, and we gathered 40 data set using hp laptop with 900 pixels of resolution and also from mobile camera Samsung note 4 with 2460 $\mathrm{x} 1440$ of resolution. As a result of a restriction in this system, the position of the facial image must be in the middle several conditions also was adding of image testing to be tested in a system, which was created the experiment was implemented on intel cori3-8100 processor, 3.60 GHz using Matlab program version 2015. The result of our proposed method showing in the following Figure 3.
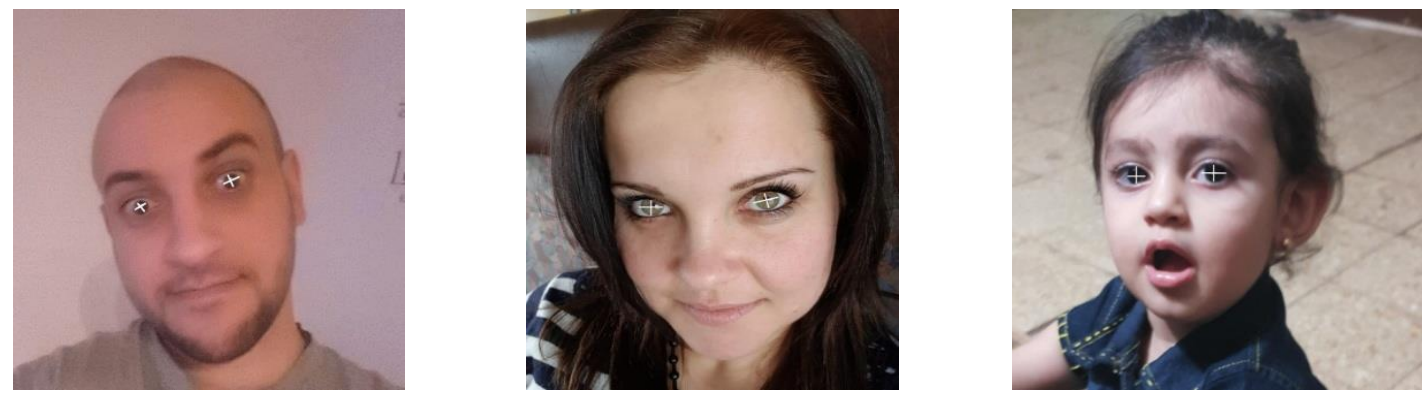

Figure 3. Examples of detected eyes by the proposed method

The percentage of the successful prediction (PSP) is used to calculate the results that were obtain from the experimental. Calculation the Accuracy to measuring the capacity of a system that has been made for object recognizing is very important. In our experimental, the detection rate was $73.2 \%$ while false rate was $12.9 \%$ as we showing in the following Table 1 depended on the formula 5. 
Detection rate $=\frac{S P}{S P+I N}$

$\mathrm{SP}=\mathrm{Person} / \mathrm{succes}$ sful facial image and eye recognition

$\mathrm{IN}=$ the image was not eye region, But detected as an eye

$$
\text { Accuracy }=\frac{S P+I N}{\text { total data }} \times 100 \%
$$

Table 1. The Result of eye Detection for our Proposed Method

\begin{tabular}{llll}
\hline Method & False rate & Detection Rate & Time of Processing \\
\hline Image based method & $20 \%$ & $68.3 \%$ & $3.2 \mathrm{~s}$ \\
Proposed method & $10.2 \%$ & $72.5 \%$ & $2.8 \mathrm{~s}$ \\
\hline
\end{tabular}

In our Article we also applied the same data set for different algorithm as showing in the following Table 2 to compare the result among them the first was Haar like features (Viola and Jones's) [18], based on Haar-like features Used for face detection. Haar-like features Encodes a contrast between the vector regions in the image. These set of the features it's can be used for Encoding Contrast Displayed by special relationships of the human face.

Neural Networks [19] artificial neural networks are able to store knowledge gained in problem solving, Thus acquiring new knowledge through experience. The systems mimic brain structure, be able to develop so-called intelligence, Using the ability of computers to learn through errors.

Support Vector Machines [20], the basic idea behind the SVM is to find, the surface of the optimal linear resolution on a conceptual basis of Reducing structural risks. The surface of the decision is a weighted combination of training group elements. These items are called support vectors and the boundary between the two classes is distinguished [21].

Table 2. Comparis on Results

\begin{tabular}{llll}
\hline No & Method & Detection rate & False rate \\
\hline 1 & Viola and Jones's & $92.3 \%$ & $3 \%$ \\
2 & Neural Networks & $88.1 \%$ & $8.7 \%$ \\
3 & Support Vector Machines & $89.3 \%$ & $10 \%$ \\
4 & Proposed method & $86.6 \%$ & $11.8 \%$ \\
\hline
\end{tabular}

\section{CONCLUSIONS}

Because no analysis has been performed on other human face images banks, the following conclusions are likely to be closely related to the bank used, mainly with the size of the images of the faces and with the type of illumination used to obtain them.

The segmentation technique used to extract the regions of interest behaved relatively well, especially in the images that had frontal illumination. It was verified that the detection of valleys made on the $\mathrm{R}$ channel presented better performance than the other three versions ( $\mathrm{G}, \mathrm{B}$, and gray levels) and that the main responsible for the overlap of the regions of interest was the Gchannel, the regions of the eyes with the regions of the eyebrows. It is believed that in the next experiment a better result can be obtained by making the (or logical) union between the (binary) images of the $\mathrm{R}$ and $\mathrm{B}$ channels. The results obtained with the use of the variance were very stimulating, it was observed that this dispersion measure has great discriminatory power for the task of detecting eyes, which encourages the search for new types of windows (portions) that best represent the region of the eye or, alternatively, to associate another measure that reflects some kind of spatial relationship between the detected regions, as, for example, measures of symmetry with respect to the main axis of the face.

\section{REFERENCES}

[1] Craw, Ian, David Tock, and Alan Bennett. "Finding face features." In European Conference on Computer Vision, vp. 92-96. Springer. Berlin. Heidelberg. 1992.

[2] Nasiri, Jalal Aldin, Sara Khanchi, and Hamid Reza Pourreza. "Eye detection algorithm on facial color images." In Modeling \& Simulation, 2008. AICMS 08. Second Asia International Conference on, pp. 344-349. IEEE, 2008.

[3] Graf, Hans Peter. "Locating faces and facial parts." In Proc. International Workshop on Automatic Face-and Gesture-Recognition, pp. 41-46. 1995. 
[4] Daway, Hazim G., and Ahmed Rafid Hashim. "Pupil Detection Based on Color Difference and Circular Hough Transform." International Journal of Electrical and Computer Engineering (IJECE) 8, no. 5 (2018).

[5] Feng, Guo Can, and Pong C. Yuen. "Multi-cues eye detection on gray intensity image." Pattern recognition 34, no. 5 (2001): 1033-1046.

[6] Huang, Weimin, and Robert Mariani. "Face detection and precise eyes location." In Pattern Recognition, 2000. Proceedings. 15th International Conference on, vol. 4, pp. 722-727. IEEE, 2000.

[7] Huang, Jeffrey, and Harry Wechsler. "Eye detection using optimal wavelet packets and radial basis functions (rbfs)." International Journal of Pattern Recognition and Artificial Intelligence 13, no. 07 (1999): 1009-1025.

[8] Pentland. Alex. Baback Moghaddam, and Thad Starner. "View-based and modular eigenspaces for face recognition." (1994).

[9] Sirohey, Saad A., and Azriel Rosenfeld. "Eye detection in a face image using linear and nonlinear filters." Pattern recognition 34, no. 7 (2001): 1367-1391.

[10] Kawato, Shinjiro, and Nobuii Tetsutani. "Detection and tracking of eyes for gaze-camera control." Image and Vision Computing 22. no. 12 (2004): 1031-1038.

[11] Kumar, R. Thilak, S. Kumar Raja, and A. G. Ramakrishnan. "Eve detection using color cues and projection functions." In Image Processing. 2002. Proceedings. 2002 International Conference on, vol. 3, pp. III-III. IEEE, 2002.

[12] Utaminingrum, Fitri, Renaldi Primaswara Praetya, and Yuita Arum Sari. "Image Processing for Rapidly Eye Detection based on Robust Haar Sliding Window." International Journal of Electrical and Computer Engineering (IJECE) 7, no. 2 (2017): 823-830.

[13] Biswas, Rubel, Jia Uddin, and Md Junayed Hasan. "A New Approach of Iris Detection and Recognition." International Journal of Electrical and Computer Engineering (IJECE) 7, no. 5 (2017): 2530-2536.

[14] Khellah, Fakhry M. "Texture classification using dominant neighborhood structure." IEEE Transactions on image processing 20. no. 11 (2011): 3270-3279.

[15] Uddin, Jia, Myeongsu Kang, Dinh V. Nguyen, and Jong-Myon Kim. "Reliable fault classification of induction motors using texture feature extraction and a multiclass support vector machine." Mathematical Problems in Engineering 2014 (2014).

[16] Ito, Yasutaka, Wataru Ohyama, Tetsushi Wakabayashi, and Fumitaka Kimura. "Detection of eyes by circular Hough transform and histogram of gradient." In Pattern Recognition (ICPR), 2012 21st International Conference on, pp. 1795-1798. IEEE, 2012.

[17] Maragos, Petros. "Tutorial on advances in morphological image processing and analysis." Optical engineering 26, no. 7 (1987): 267623.

[18] Viola, Paul, and Michael J. Jones. "Robust real-time object detection. Cambridge Research Laboratory." Tech Rep: CRL(2001).

[19] Lu, Huchuan, Wei Zhang, and Deli Yang. "Eye detection based on rectangle features and pixel-pattern-based texture features." In Intelligent Signal Processing and Communication Systems, 2007. ISPACS 2007. International Symposium on, pp. 746-749. IEEE, 2007.

[20] Jee, Hyungkeun, Kyunghee Lee, and Sungbum Pan. "Eye and face detection using SVM." In Intelligent Sensors, Sensor Networks and Information Processing Conference, 2004. Proceedings of the 2004, pp. 577-580. IEEE, 2004.

[21] Kim. Hvoung-Joon. and Whoi-Yul Kim. "Eye detection in facial images using Zernike moments with SVM." ETRI journal 30, no. 2 (2008): 335-337.

[22] Lin, Zhui, Lide Wang, Jieqiong Zhou, and Liyuan Liu. "Efficient Train Driver Drowsiness Detection on Machine Vision Algorithms." Indonesian Journal of Electrical Engineering and Computer Science 11, no. 5 (2013): 25662573.

[23] Kiran, P., H. S. Mohana, and P. A. Vijaya. "Human machine interface based on eye wink detection." International Journal of Informatics and Communication Technology (IJ-ICT) 2, no. 2 (2013): 116-123. 